\title{
Identification of the state of the soil-plant systems on the RUDN-University campus (based on PAH concentrations)
}

\author{
Aleksandr Khaustov $^{1, *}$, Margarita Redina ${ }^{1}$, Zhandos Kenzhin $^{1}$, Dmitry Gabov ${ }^{2}$ and \\ Evgeniya Yakovleva ${ }^{2}$ \\ ${ }^{1}$ Peoples' Friendship University of Russia (RUDN University), Faculty of Ecology, 6 Miklukho- \\ Maklaya St, Moscow, 117198, Russian Federation \\ ${ }^{2}$ Institute of Biology of Komi Science Centre of the Ural Branch of the Russian Academy of Sciences, \\ 28 Kommunisticheskaya st., 167982 Syktyvkar, Komi Republic, Russia
}

\begin{abstract}
The environmental state of soil-plant systems under the condition of technogenic pressure has been demonstrated on the example of the social significant territory in the city of Moscow - the campus of the RUDN University. The assessment is based on the analyses of the environmental fate of polycyclic aromatic hydrocarbons (PAH) in soil, roots and aerial parts of plants of bluegrass meadow: Naphthalene (Naph), Fluorene (Fluorene), Phenanthrene (Phen), Anthracene (An), Fluoranthene (Flu), Pyrene (Py), Chrysene (Chr), Benzo[a]anthracene (BaA), Benzo[a]pyrene (BaP), Benzo[b]fluoranthene (BbFlu), Benzo[k]fluoranthene (BkFlu), Benzo[ghi]perylene (Bghi), Indeno[1,2,3cd[pyrene (IP), Dibenzo[a,h]anthracene (DBa). These pollutants are considered as geochemical markers and reflect quite detailed the integral state of soil-plant systems. As a result, the zones with relative prosperous and with critical state are identified. The connection between physicochemical characteristics $\left(\log \mathrm{K}_{\mathrm{ow}}\right)$ of pollutants and their accumulation activity (concentration coefficients) is confirmed.
\end{abstract}

\section{Introduction}

Urban soil-plant systems are objects of a whole range of technogenic impacts due to the activity, primarily, of industrial and transport pollution sources. Under Moscow conditions, it is transport loads that prevail in the formation of environmental pollution. However, the influence zones of the transport routes depend both on the actual activity of the transport and on the specifics of the polluted systems and the characteristics of the emission components. In this regard, local pollution models are of great scientific interest and are characterized by significant diversity. To identify the zones of influence of technogenic sources, it is necessary to apply the reliable markers that allow to clearly attribute the studied object to a certain type of pollution

Plants play an important role in the accumulation and conversion of pollutants

\footnotetext{
*Corresponding author: khaustov-ap@ @rudn.ru
} 
(including hydrocarbons) in environmental components. This became the basis for the development of methods and technologies for phytoremediation. The experiments [4] established a positive effect of plants on soil cleaning, but it is believed that the effect of using plants is low with respect to PAHs - a decrease in polyarenes of $0.8-14$ times depending on the experiment time, types of vegetation, and concentrations of PAHs introduced. The greatest effect was recorded for pyrene and phenanthrene during twomonth observation of remediation by various plants. For all types of phytoremediation, which leads to a natural decrease in PAHs in soils (phytoextraction, phytofiltration, phytovolatilization, rhizoremediation, phytostabilization, phytodegradation), their assimilation (sorption) by vegetation is necessary. The main processes leading to the penetration of hydrocarbons into plant organisms are phytofiltration and rhizomediation, which control the movement (albeit weak due to the high hydrophobicity of hydrocarbons) of solutions in the soil - root - stem system [4]. A significant role is given to the destruction of PAHs in the rhizosphere with the participation of microbial communities, that is, plants themselves are only a part of the general system of PAH decomposition in soils $[2,6]$. When predicting these processes, it is necessary to take into account the heterogeneity of hydrocarbons distribution in the soil, the hydrophobicity of pollutants, the growth of vegetation, the possibility of PAHs coming from the atmosphere into plants, as well as the natural generation of PAHs by plant organisms themselves. The role of these factors for soil-plant systems with different levels of technogenic loads can be redistributed. Thus, the property of hydrophobicity of PAH compounds (expressed by the distribution coefficient in the octanol-water system $\log K_{O w}$ ) does not always uniquely determine the behavior of PAHs in the environment due to the specificity of technogenic pressure on these media. Let us demonstrate this by the example of soil-plant systems on the campus of the Peoples' Friendship University of Russia.

\section{Research methods and objects}

The object of research in this article is soil-plant systems on the campus of the Peoples' Friendship University of Russia. The territory is located in a relatively prosperous area of Moscow, however, a major transport highway (Leninsky Prospekt) and streets quite loaded with vehicles are adjacent to it. Since 2017, environmental monitoring of the campus has been organized, covering all environmental components. As of today, about 4000 determinations of pollutants in air, snow cover, soil, vegetation have been carried out at 33 points of the monitoring network; acoustic load levels, electromagnetic fields and radiation background are estimated. These works provide reliable and complete assessments of the state of the environment.

Particular attention is paid to the identification of marker substances and priority pollutants in the environmental components. PAHs, which are geochemical markers of natural and man-made processes, can be represented as such compounds [1, 2]. PAH molecules contain 2 or more aromatic rings in their structure, have a relatively high resistance in the environment and low solubility in water, are lipophilic and can cause mutagenic and carcinogenic effects. Analysis of PAH concentrations in environmental objects allows to associate pollution to a certain source: a natural or man-made process that occurs at elevated temperatures or under "normal" conditions. Most often, for the purpose of identifying sources, indicator ratios of PAH concentrations are used: the ratio of 2-3-ring to 4-5-ring sums, or the ratio of isomer concentrations within a single molecular weight.

For the purpose of this study, we analyzed the contents of 14 PAHs in the soils, roots, and aerial parts of plants of meadow bluegrass (Poa praténsis) on campus. The analysis was carried out at the Institute of Biology, Komi Scientific Center, Ural Branch of the Russian Academy of Sciences. A detailed description of the technique is presented in [2]. 
Previously, on the basis of our earlier observations, we divided the territory into a conditionally clean zone (a "background" zone in a forest park, a recreation zone) and a territory with an increased level of pressure. An analysis of the spatial distribution of PAHs in soils, roots, and aerial parts of plants allows a more detailed assessment of the area of influence of vehicles on the territory. First of all, estimates of PAH migration in the soil root - stem chain were carried out: - concentration coefficients (CC) were calculated as the ratio of PAH concentration in the "receiving" medium to the concentration in the "giving" one. With QC> 1, PAH transition is claimed. CC calculations were carried based on the PAH analysis data from 33 monitoring points; then estimates of PAH migration activity for various campus areas were obtained. Based on the $\mathrm{CC}$ values for the identified individual PAHs, using the cluster analysis procedure (Ward's method, Euclidean distances), the "soil - root" and "root - stem" medium borders were classified. This allows us to compare the activity of PAHs destruction under the influence of the "rhizosphere effect" - to correlate the activity of destruction under the influence of microorganisms living in the rhizosphere to the activity of this process without the participation of microorganisms, by the "forces" of plants alone. As a rule, such affected areas with minimal activity of microbiota are identified in the immediate vicinity of the loaded traffic routes. The differences in these environments are determined by the fact that the number of hydrocarbon-oxidizing bacteria in the rhizosphere is several times higher than in soils without plants.

The self-purification mechanisms of soils are of a staged nature, associated with the seasonal development of vegetation and, accordingly, soils, which are considered as a medium for a bacterial filter that retains and transforms PAHs. According to published data, the bioconcentration of PAHs by vegetation from soils varies from $10^{-6}$ to 16 , and for individual polyarenes it can reach 30-40 [4].

The most important characteristic of PAHs, which determines the specificity of their behavior in relation to organisms, is $\log \mathrm{K}_{\mathrm{ow}}$. This indicator characterizes not only the hydrophobicity (lipophilicity) of the molecules themselves, but also the ability of substances to bioaccumulate. This property is considered expressed when log Kow> 3; with a value above 4 , the substance is classified as of an increased hazard class and a high tendency to bioaccumulation is predicted for such a compound [5]. All PAH compounds (except Naph) have a $\log \mathrm{K}_{\mathrm{ow}}$ of more than 3.2, and starting with 4-ring ones - and higher than 4 . In connection with this, the $\log \mathrm{K}_{\mathrm{ow}}$ indicator was given special attention in the study: we assume that the correlation between log Kow and CC substances should testify to the activity of PAH movement processes in soil-plant systems and the levels of technogenic loads on them.

The revealed patterns of PAH distribution were visualized based on the Surfer GIS package.

\section{Results and discussion}

The groups of borders-barriers in soil-plant systems identified using cluster analysis are presented in Table. 1.

The features of the first group include the active transition of light PAHs (2-3 benzene rings) at extremely low values for the sum of PAHs in general. These are mainly borders in the soil-root system, as well as root-stem borders at the borders of the background or lightly pressured territory, but not in the directly roadside parts.

In cluster 2, PAH migration can be characterized as nonequilibrium technogenic. PAH flows in the soil - root system are weakly expressed, and the stem part is polluted from the atmosphere mainly by multi-ring PAHs due to the combustion products of motor fuel. This is confirmed by calculations of the ratios of kinetic to thermodynamic polyarenes. Point 27 is far enough from the main highway, however, it is located within the car parking of the 
RUDN University employees.

Table 1. Characterization of HCB clusters on campus in terms of migration with the accumulation of PAHs

\begin{tabular}{|c|c|c|c|}
\hline Cluster & Borders & $\begin{array}{l}\text { CC of } \\
\Sigma \text { PAH }\end{array}$ & $\begin{array}{c}\text { Individual PAHs with maximum } \\
\text { CC }\end{array}$ \\
\hline 1 & $\begin{array}{l}\text { Technogenic migration under the } \\
\text { conditions of saturation of } \\
\text { environments }\end{array}$ & $\frac{0.02-3.35}{1.09}$ & Fluorene, Naph, Phen, An \\
\hline 1.1 & Active transition is unlikely & $\frac{0.02-1.09}{0.65}$ & Fluorene, Phen, Naph \\
\hline 1.1 .1 & $\begin{array}{l}\text { Soil-roots on the points } 10,11,16 \\
17,21,22,25,26,29,31\end{array}$ & $\frac{0.02-0.86}{0.52}$ & Fluorene, Naph, Phen \\
\hline 1.1 .2 & Soil-roots on the points 4,27 & $\frac{0.73-1.09}{0.87}$ & Phen, (Fluorene, An) \\
\hline 1.2 & Transition is real & $\frac{0.63-3.35}{1.44}$ & Fluorene, Naph, Phen, An, Ip \\
\hline 1.2 .1 & $\begin{array}{c}\text { Soil-roots on the points } 7,12,24 \\
\text { root-stem on the points } 5,8,9,13 \\
15,17,19,23,25,27,28,29,30\end{array}$ & $\frac{0.63-1.60}{1.10}$ & Fluorene, Phen, Naph, An \\
\hline 1.2 .2 & $\begin{array}{l}\text { Soil-roots on the points } 1,32 ; \\
\text { root-stem on the points } 2,11,14,22\end{array}$ & $\frac{0.63-3.35}{1.90}$ & $\begin{array}{c}\text { Fluorene, Naph, Phen, An, IP, Chr, } \\
\text { Flu=Py }\end{array}$ \\
\hline 2 & Ative technogenic migration & $\frac{0.54-3.51}{1.65}$ & $\begin{array}{l}\text { Bghi, An, Fluorene, BkFlu, Chr, } \\
\text { Phen, Py, Naph, Flu, BbFlu, BaA } \\
\text { (Bap, DbA) }\end{array}$ \\
\hline 2.1 & $\begin{array}{c}\text { Weakly expressed technogenic } \\
\text { migration }\end{array}$ & $\frac{0.54-3.16}{1.51}$ & $\begin{array}{l}\text { Bghi, Chr, Phen, An, BkFlu, } \\
\text { Fluorene, Flu, Py, BbFlu, Naph, } \\
\text { (Bap, DbA) }\end{array}$ \\
\hline 2.1 .1 & $\begin{array}{l}\text { Soil-roots on the points } 5,6,15,18 \text {, } \\
\qquad 1923\end{array}$ & $\frac{0.69-3.16}{1.85}$ & $\begin{array}{l}\text { Bghi, BbFlu, BkFlu, BaA, Chr, } \\
\text { Phen, Flu, An, BaP, Fluorene, } \\
\text { DbA=Naph=Py, (IP) }\end{array}$ \\
\hline 2.1 .2 & $\begin{array}{l}\text { Soil-roots on the points } 2,8,14,20 \text {, } \\
28,30,33\end{array}$ & $\frac{0.54-2.96}{1.34}$ & $\begin{array}{l}\text { Chr, Phen, An, Fluorene, BkFlu, } \\
\text { Py, Flu, Naph, (DbA, BaA, BbFlu) }\end{array}$ \\
\hline 2.2 & $\begin{array}{l}\text { Intensive manifested technogenic } \\
\text { migration }\end{array}$ & $\frac{1.15-3.51}{2.58}$ & $\begin{array}{l}\text { An, Fluorene, BkFlu, Naph, Py, } \\
\text { Chr, Phen, Flu, BbFlu, (BaA, BaP) }\end{array}$ \\
\hline 2.2 .1 & $\begin{array}{l}\text { Soil-roots on the points } 3 ; 20 \\
\text { root-stem on the points } 3,12\end{array}$ & $\frac{3.09-3.51}{3.30}$ & $\begin{array}{l}\text { An, Fluorene, Naph, Py, Flu, } \\
\text { BbFlu, Chr, Phen, BkFlu, (BaP, } \\
\text { BaA) }\end{array}$ \\
\hline 2.2 .2 & $\begin{array}{l}\text { Soil-roots on the points } 9,13 \\
\text { root-stem on the points } 1,4,6,7 \text {, } \\
10,16,18,20,21,24,26,31,33\end{array}$ & $\frac{1.15-2.58}{1.86}$ & $\begin{array}{l}\text { BkFlu, Fluorene, Chr, Phen, } \\
\text { Naph= Py, Flu, An (BaP) }\end{array}$ \\
\hline
\end{tabular}

The low probability of an active transition in cluster 1.1 indicates the loss of selfcleaning functions of PAHs by the soil layer. Here, the maximum saturation of the rhizosphere with polyarenes is typical, which prevents their entry into the stems. High PAHs toxicity does not allow the microbiota to form favorable conditions for the promotion of nutrients in the stems. Despite regular watering of the highway and the surrounding area, the soils do not manage to form nutrients that can support a stable vegetation cover.

For a real transition (cluster 1.2), CC $>1$ is recorded at many points in the transition zone between Leninsky Prospekt and Miklukho-Maklaya street, where vegetation forms flows from the root to the stems and, naturally, from the soil to the roots. These are 2-3 ring PAHs with possible self-generation, but mostly man-made. The cluster covers a forest Park zone with the maximum potential for self-cleaning of soil and vegetation in natural conditions. When the rains fall, certain plant species experience man-made pressure. 
Further analysis of the distribution of PAHs in the background territory and in the zone with increased transport load confirmed the correctness of the allocation of this group: regardless of the level of anthropogenic load, it is in the soil-root system that the flows of PAHs are maximally ordered. This border turns out to be weakly permeable for heavy PAHs, and the CC for the total amounts of PAHs is relatively small.

The second group of borders is maximally permeable for a large set of individual PAHs; the $\mathrm{CC}$ values are generally higher than in the first group. Most of the borders here are the "rootstem" at points in the zone of direct influence of the roads. Obviously, this is precisely what determines the diversity of the association of migrating PAHs (Table 1): there is contact with air polluted by vehicles. However, it was not possible to detect "heredity" in the intensity of PAHs migration at the observation points. Table 2 shows the numbers of points that are characterized by a complete absence of PAHs transition, minor CC or pronounced transition. Most likely, more factors affect the conditions of PAHs entry and accumulation in the aerial part than in the case of migration in the soil-plant system. At a minimum, it should be noted the probability of introducing PAHs with air flows and the processes of PAHs degradation due to the photolysis. As a result, the distribution of PAHs in the ground parts of plants may be more "blurred".

Table 2. PAH transition activity characteristics

(taking into account the probability of determination error of $30 \%$ )

\begin{tabular}{|c|c|c|c|}
\hline \multirow{2}{*}{$\begin{array}{c}\text { Transition } \\
\text { border }\end{array}$} & \multicolumn{3}{|c|}{ Numbers of points where the transition is... } \\
\hline & $\begin{array}{c}\text { absent } \\
(\mathbf{K K}<0.7)\end{array}$ & $\begin{array}{c}\text { possible } \\
(\mathrm{KK}=\mathbf{0 . 7 - 1 . 3 )}\end{array}$ & $\begin{array}{c}\text { mannifested } \\
(\mathbf{K} \mathbf{>}>\mathbf{1 . 3})\end{array}$ \\
\hline Soil - root & $\begin{array}{l}8,9,13,14 \\
20,28,30,33\end{array}$ & $\begin{array}{l}2,3,5,6,10,15,18,19 \\
22,23,24,26,29\end{array}$ & $\begin{array}{l}1,4,7,11,12,16,17,21,25,27,31,32 \\
\mathrm{CC}_{\max }=2.74 ; \mathrm{CC}_{\text {average }}=1.72\end{array}$ \\
\hline Root - stem & 1,10 & $\begin{array}{l}3,4,6,7,12,13,15,16 \\
17,18,20,21,23,24,26 \\
27,28,29,31,32,33\end{array}$ & $\begin{array}{l}2,5,8,9,11,14,19,22,25,30 \\
\mathrm{CC}_{\max }=3.51 ; \mathrm{CC}_{\text {average }}=2.15\end{array}$ \\
\hline
\end{tabular}

When comparing the activity of polyarene migration in the soil - root and root - stem systems (Table 1), it is impossible to distinguish "unambiguously active" zones of the campus, where both PAH transitions would be equally intense. Of all the points examined, only on the points 11 and 25 significant CCs were noted for both systems (1.5 - 1.9 in both cases: significant, but not maximum CCs). In all other cases, the activity of PAHs migration from soils to the roots and from roots to the aerial parts of plants is different (Fig. 1).

Maps show significantly different conditions for PAH migration in the background and transport-loaded zones. This may be due to the fact that intense pollution has already led to the accumulation of significant amounts of PAHs in all the environmental components. Soils, unlike plants with their seasonality of life processes (especially for annual plants, as in our case), are more prone to PAH deposition. Therefore, the processes of migration and accumulation of PAHs in the soil-root system look more streamlined, and the very ability to take new "portions" of PAHs in vegetation is higher - and due to the greater "capacity" (smaller amounts of PAHs are initially accumulated and there are opportunities to skip larger masses of PAHs across the border), and due to large sources of input (both from the soil through the root systems, and from the atmosphere due to transport). 

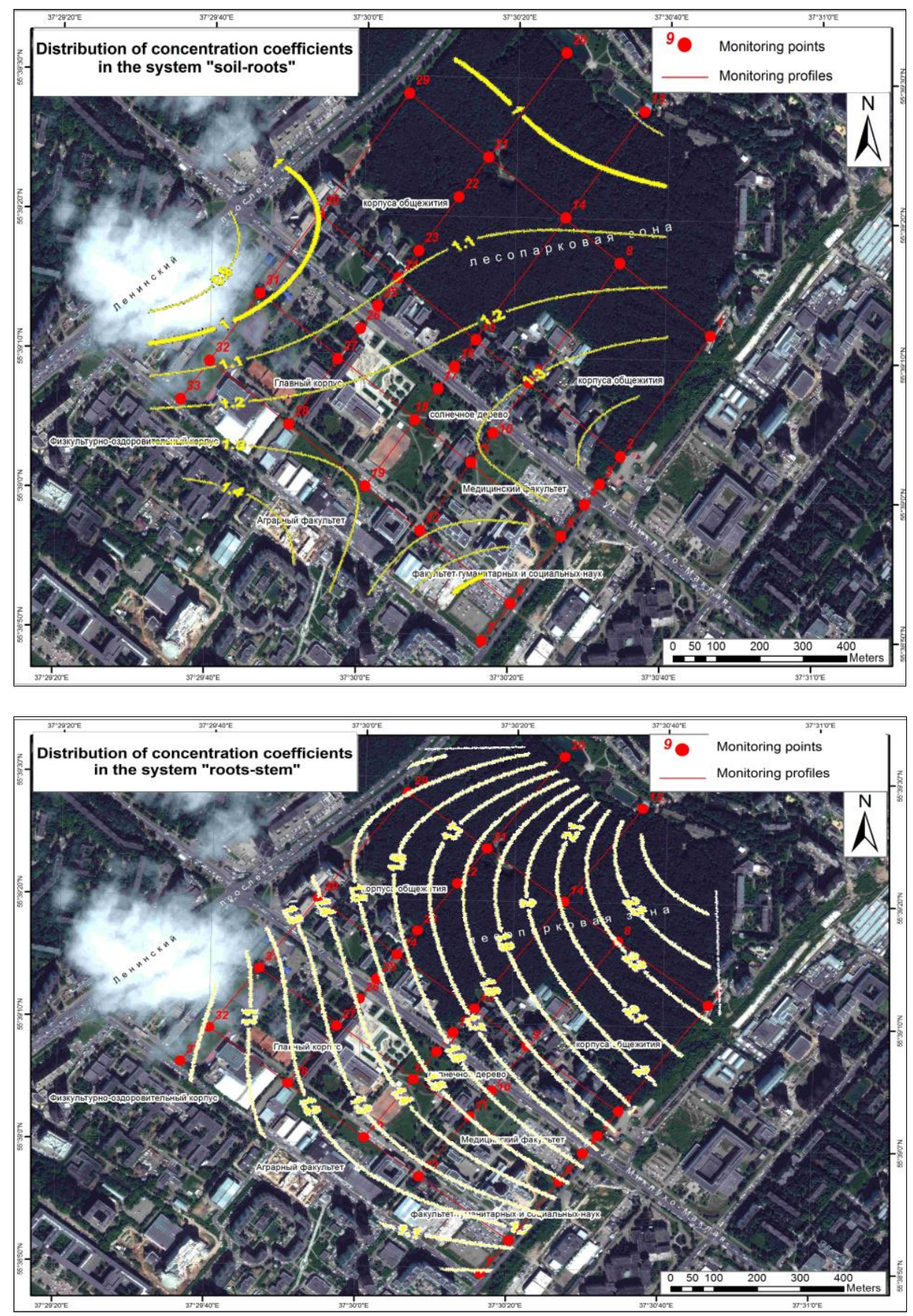

Fig. 1. CC of PAHs in the systems "soil-root" (upper) and "root-stem" (lower)

An analysis of the relationship between log Kow and CC for the "soil - root" and "root - stem" systems (Fig. 2) shows differences in the state of soil - plant systems due to the uneven technogenic loads. 

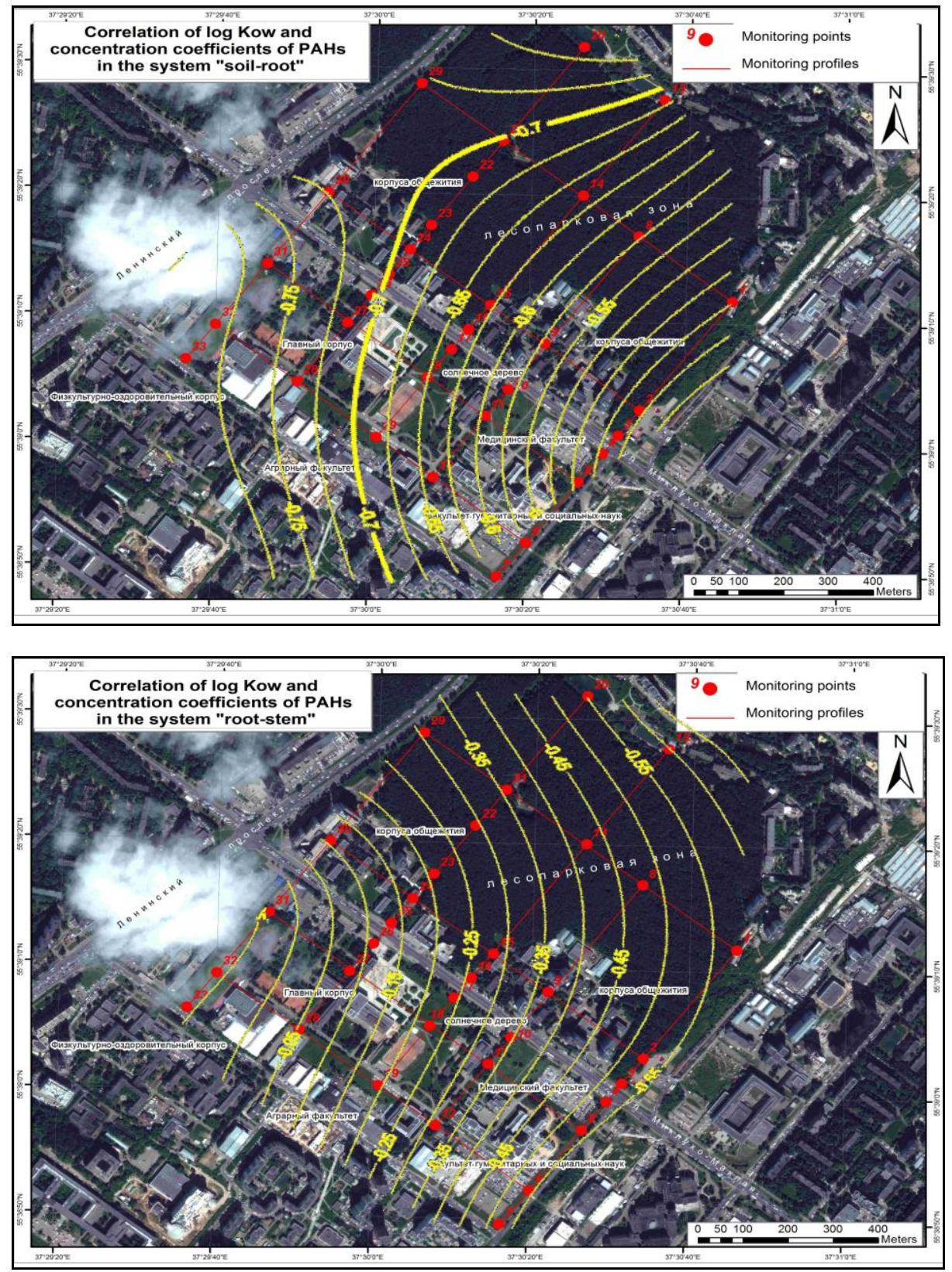

Fig. 2. Correlations between $\log \mathrm{K}_{\mathrm{ow}}$ and CC of PAHs in the "soil-root" (upper) and "root-stem" (lower) systems

As can be seen, in contrast to clearly defined negative relationships for the soil - root system, in the case of the root - stem system, the correlations of log Kow with CC are not so obvious. In some cases, these are insignificant positive connections (vols. 2, 3, 14, 15, 18,19 ); but for most points, negative or close to missing points. Such a variety can testify to the significant role of PAHs aerogenic supply to the plant organism. In addition, for points with a positive correlation (14 is the background; 2, 3, 15, 18, 9 are the technogenic load below the maximums), we can assume the natural generation of PAHs. 


\section{Conclusion}

Roots play the role of "filters", creating natural barriers to the separation of PAHs, both in the background and transport-pressured zones. Anthropogenic load plays an unconditional role in the processes of PAH fractionation and determines the initial PAH masses that "claim" to be transferred. However, the composition of associations that penetrate the "soil - root" border and the transition intensity themselves are generally similar: 2-3 ring PAHs migrate relatively easily. QCS for larger molecules may be an order of magnitude smaller or even beyond the scope of significance.

$\mathrm{CC}$ in the background zone is clearly higher compared to the $\mathrm{CC}$ of polyarenes in the transport loaded zone A possible explanation is that "saturation" of the "host environment" has been achieved and more active PAH transitions do not occur even under conditions of much higher levels of soil contamination compared to the background.

In root - stem systems, patterns are not so obvious in the background territory. You can select the maximum CC coefficients at point 14 (except for BbFlu and heavy PAHs), followed by point 22 . In the transport-loaded zone, uniform transitions of the entire PAH Association are characteristic; peaks are at point $23(\mathrm{An})$ and 11 (BbFlu).

The obvious dependence of PAH accumulation processes in the root system when received from soils with a $\log \mathrm{K}_{\mathrm{ow}}$ coefficient was confirmed. However, the spatial distribution of the correlation index of this coefficient with the activity of PAHs uptake by roots and subsequent transport to the aerial part indicate that the anthropogenic load has a significant impact on these processes. The correlations are less pronounced in areas with a strong influence of transport pollution. Obviously, the state of the oppressed microbiota in the rhizosphere is also manifested here - it ceases to prevent the entry of PAHs into plant organisms and there is a more active absorption of PAHs by them.

\section{References}

1. A. P. Khaustov, M. M. Redina., Geochem. Int., 55(1), 98-107 (2017)

2. A. P. Khaustov, M. M. Redina., E.V. Yakovleva, Geoecology. Eng. geology, hydrogeology, geocryology, 3, 3-17 (2018) (in Russian)

3. A. P. Khaustov, M. M. Redina., E.V. Yakovleva, E3S Web of Conferences, 98, 01027 (EDP Sciences, Les Ulis, 2019)

4. A. A. Oborin, Oil polluted biocenoses (formation processes, scientific bases of recovery, medical and ecological problems) (Perm state University, Perm, 2008) (in Russian)

5. OECD Environment, Health and Safety Publications, Series on Testing and Assessment, No. 27, (Environment Directorate, Organization for Economic Cooperation and Development, 2001)

6. E. V. Yakovleva, D. N. Gabov, V. A.Beznosikov et al., Polycyclic Aromatic Compounds, 37(2-3), 203-218 (2017) 